\title{
Modernidad y xenofobia en Andalucía y Cataluña: un análisis comparativo
}

\author{
Gonzalo Herranz de Rafael \\ Universidad de Almería \\ gherranz@ual.es
}

Recepción: 24/07/2008

Aceptación: 15/06/2009

\section{Resumen}

Esta investigación es un estudio cuantitativo sobre la xenofobia en la primera década del siglo XXI. Especialmente, analiza de manera comparativa, a partir de datos primarios procedentes de encuestas, el índice de xenofobia existente en dos comunidades autónomas: Andalucía y Cataluña, regiones que se encuentran en dos estadios diferentes de modernización. A partir de tres modelos de regresión múltiple, se ha pretendido analizar el índice de xenofobia en ambas comunidades y, como consecuencia, se ha observado que la modernidad es un buen disolvente de la xenofobia.

Palabras clave: xenofobia, modernización, inmigración, estudio cuantitativo, Andalucía, Cataluña.

Abstract . Modernity and xenophobia in Andalusia and Catalonia: A comparative analysis

This article represents a quantitative study on xenophobia during the first decade of the current millenium. It comparatively analyses the level of xenophobia that exists in two Spanish Autonomous Communities - Andalusia and Catalonia - which are at different stages of modernisation. Three models of Pearson's correlations were used in order to analyse the level of xenophobia in both regions, and it was observed that modernity is a good solvent of xenophobia.

Key words: xenophobia, modernisation, immigration, quantitative study, Andalusia, Catalonia.

\section{Sumario}
1. Introducción y limitación de objetivos
4. Hipótesis y datos
2. Sobre el concepto de xenofobia
5. Análisis de los resultados
y racismo
6. Conclusiones
3. El concepto de densidad
7. Referencias bibliográficas 


\section{Introducción y limitación de objetivos}

Este artículo examina de forma comparada la xenofobia existente en dos comunidades autónomas con diferentes niveles de modernización, como son Andalucía y Cataluña. Además, se ha creído conveniente analizarla a partir de los niveles de densidad poblacional existentes a nivel autonómico.

La reciente concentración de inmigrados en España, y particularmente en determinadas zonas de Andalucía y Cataluña, es suficientemente significativa para justificar la necesidad de analizar los posibles efectos que conlleva la mayor o menor densidad de inmigrantes en los distintos contextos de recepción. De hecho, a 1 de enero de 2007, el total de residentes en España era de 45.200.737 personas, de las cuales 4.519.554 eran extranjeras, cifra que representaba al $10 \%$ del total de la población. Sin embargo, a finales de los años noventa, el porcentaje no llegaba al $2 \%$, lo que significa que en menos de una década la población se ha quintuplicado. Una de las principales características de esa población inmigrada es su distribución desigual por todo el país. Las mayores concentraciones y densidades se encuentran en Madrid, Barcelona, la costa mediterránea y Canarias, frente a las comunidades de interior (Extremadura, La Rioja o Asturias), que concentran menos población extranjera. A su vez, dentro de estas zonas, los inmigrantes también siguen una distribución desigual a nivel provincial, puesto que se concentran en mayor medida en unos municipios $y$, dentro de éstos, en determinados barrios o zonas ${ }^{1}$.

Centrándonos en las dos comunidades a analizar, diremos que, aunque Andalucía tiene un mayor índice de población, que representa el 17,83\% del

Tabla 1. Extranjeros en Andalucía y Cataluña (padrón de 2007)

\begin{tabular}{lrr}
\hline & Andalucía & Cataluña \\
\hline Total extranjeros & 531.827 & 972.507 \\
Europa comunitaria & 252.726 & 230.705 \\
Resto de Europa & 25.643 & 43.547 \\
África & 110.892 & 253.016 \\
Iberoamérica & 120.639 & 352.328 \\
América del Norte & 5.792 & 5.379 \\
Asia & 15.842 & 87.028 \\
Oceanía & 261 & 456 \\
Apátridas & 32 & 48 \\
\hline Total población & $\mathbf{8 . 0 5 9 . 4 6 1}$ & $\mathbf{6 . 2 1 0 . 5 0 8}$ \\
\% de extranjeros & $\mathbf{6 , 6}$ & $\mathbf{1 3 , 4 8}$ \\
\hline
\end{tabular}

Fuente: INE. Elaboración propia.

1. Por poner un ejemplo significativo, en el caso de la provincia de Almería, según el Padrón Municipal del 2006, el porcentaje de extranjeros extracomunitarios, que son inicialmente quienes despiertan un mayor nivel de xenofobia, es del 13,83\%. No obstante, hay municipios, como Nijar, donde asciende hasta el 29,74, mientras que en algunos barrios de la población de El Ejido se sitúan en el 52,9\%. 
Tabla 2. Extranjeros en Andalucía por provincias (padrón de 2007)

\begin{tabular}{lrrrrrrrr}
\hline & Almería & \multicolumn{2}{c}{ Cádiz } & Córdoba Granada & Huelva & Jaén & Málaga & Sevilla \\
\hline Total extranjeros & 104.441 & 33.247 & 15.587 & 44.861 & 26.183 & 13.005 & 191.822 & 45.802 \\
\hline Europa comunitari** & 21.386 & 13.252 & 1.782 & 12.190 & 4.860 & 1.253 & 101.430 & 7.964 \\
Resto de Europa & 26.335 & 3.372 & 5.029 & 9.444 & 9.954 & 2.576 & 22.243 & 8.738 \\
África & 38.161 & 5.868 & 2.894 & 8.546 & 6.762 & 4.891 & 21.916 & 7.821 \\
Iberoamérica & 16.854 & 8.725 & 5.087 & 12.590 & 4.137 & 3.383 & 36.692 & 17.657 \\
América del Norte & 267 & 817 & 178 & 570 & 108 & 84 & 1.668 & 1.291 \\
Asia & 1.424 & 960 & 602 & 1.498 & 357 & 811 & 5.950 & 2.282 \\
Oceanía & 11 & 31 & 15 & 22 & 4 & 7 & 117 & 35 \\
Apátridas & 3 & 3 & - & 1 & 1 & - & 2 & 14 \\
\hline Total población & $\mathbf{6 4 6 . 6 3 3}$ & 1.207 .343 & 792.182 & $\mathbf{8 8 4 . 0 9 9}$ & 497.671 & $\mathbf{6 6 4 . 7 4 2}$ & 1.517 .523 & 1.849 .268 \\
\% de extranjeros & $16,15 \%$ & $2,75 \%$ & $1,96 \%$ & $5,07 \%$ & $5,26 \%$ & $1,95 \%$ & $12,64 \%$ & $2,47 \%$ \\
\hline
\end{tabular}

Fuente: INE. Elaboración propia.

* Se incluye la Europa de los 15, no la de los 27.

total, Cataluña concentra más de la mitad de extranjeros que Andalucía, aunque registre el 15,95\% de la población española. Más concretamente, hay 848.953 andaluces más, pero Cataluña acoge a 440.680 extranjeros más que Andalucía.

Esta diferenciación también se observa a nivel provincial, puesto que se dan mayores densidades de población extranjera en las provincias catalanas que en las andaluzas. Sobresalen especialmente Girona y Almería, con un $17,16 \%$ y $16,15 \%$, respectivamente.

Desde nuestro punto de vista, el análisis de la xenofobia debe estar segmentado atendiendo a la densidad de inmigración, ya que las encuestas a mues-

Tabla 3. Extranjeros en Cataluña por provincias (padrón de 2007)*

\begin{tabular}{lrrrr}
\hline & Barcelona & Gerona & Lérida & Tarragona \\
\hline Total extranjeros & 575.315 & 121.207 & 57.316 & 106.737 \\
\hline Europa comunitaria & 115.286 & 31.266 & 18.126 & 39.377 \\
Resto de Europa & 143.310 & 42.296 & 25.952 & 48.705 \\
África & 153.371 & 53.261 & 23.612 & 36.298 \\
Iberoamérica & 213.344 & 23.113 & 9.735 & 20.651 \\
América del Norte & 2.589 & 240 & 30 & 155 \\
Asia & 76.190 & 7.758 & 1.879 & 5.581 \\
Oceanía & 275 & 34 & 3 & 7 \\
Apátridas & - & - & - & - \\
Países desconocidos & 248 & 20 & 8 & 4 \\
\hline Total población & 5.332 .513 & $\mathbf{7 0 6 . 1 8 5}$ & $\mathbf{4 1 4 . 0 1 5}$ & $\mathbf{7 5 7 . 7 9 5}$ \\
\% de extranjeros & $\mathbf{1 0 , 7 8 \%}$ & $\mathbf{1 7 , 1 6 \%}$ & $\mathbf{1 2 , 8 7 \%}$ & $\mathbf{1 4 , 0 8 \%}$ \\
\hline
\end{tabular}

Fuente: IDESCAT. Elaboración propia.

* Los totales no coinciden, ya que la tabla general se basa en una fuente del INE y, por provincias, en IDESCAT. 
tras totales de la población reflejan opiniones y actitudes ante la inmigración poco reales, al asignarle el mismo peso a personas que viven en zonas con más de un $16 \%$ de extranjeros, como es el caso de Almería, que a otras donde la cifra no llega al 3\%, como es el de Pontevedra, según el padrón municipal de enero de 2007. Además, la mayoría de las citadas encuestas realizadas a la población española reflejan valoraciones positivas hacia la inmigración, dato que no se corresponde con el obtenido mediante otros estudios, tanto cuantitativos ${ }^{2}$, como cualitativos $^{3}$, en los que se ha tenido en cuenta una mayor segmentación de la población en función de la densidad de inmigrantes. En nuestro caso, analizaremos de forma segmentada la xenofobia existente por comunidades autónomas, tanto en Andalucía como en Cataluña, aún siendo conscientes de las limitaciones metodológicas que ello conlleva ${ }^{4}$.

Por tanto, el objetivo de este trabajo es observar si la xenofobia está determinada principalmente por la densidad de inmigrantes en un contexto de modernización.

Conceptualmente, podemos entender la modernización ${ }^{5}$ como un proceso continuo, a largo plazo e inacabado de cambio social. La modernidad es el

2. Véase G. Herranz (2008), «Xenofobia: un estudio comparativo en barrios y municipios almerienses», Reis, 121, 107-133. Desde una perspectiva teórica, A. Portes y R. Rumbaut (1996), Inmigrant in American, California, University of California Press; M. Zhou, «Segmented assimilations: issues, controversias and recent research on the new second generation", International Migration Review, 31 (4), 975-1008.

3. Véase, por ejemplo, el resumen que realiza González Enríquez, tanto de su trabajo como de otros estudios cualitativos sobre inmigrantes en barrios de alta densidad de extranjeros extracomunitarios y de sus efectos negativos: «Varios estudios cualitativos que han utilizado la técnica de las entrevistas a grupos y se han dirigido a las ciudades de Madrid, Barcelona, Alicante y Valencia, y dentro de ellas a los barrios con alta densidad de inmigrantes — por encima del $15 \%$ de la población total— han encontrado una actitud general, extendida y profunda, de rechazo hacia la convivencia con los inmigrantes, que se expresa con un sentimiento de haber sido "invadidos" y que se traduce en una opinión negativa global sobre la inmigración en España. El temor y las molestias causadas por la concentración en los espacios públicos - calles, plazas, parques - de individuos de otras razas o de otras costumbres, la percepción generalizada de que en esas zonas la seguridad ha disminuido a la vez que aumentaba el número de extranjeros en los bloques de pisos y en los espacios públicos entre los españoles y los extranjeros, especialmente cuando éstos viven en condiciones de hacinamiento, todo ello forma un ambiente de rechazo en el que la inmigración se vive como un problema importante para el barrio que disminuye su calidad de vida» (C. González Enríquez, Opinión pública y política de inmigración: Elementos de conflicto en la convivencia con los inmigrantes en España, Departamento de Ciencias Políticas y de la Administración, UNED, CPA Estudios, Working Papers, 9/2004, 9.

4. Por problemas metodológicos de la asignación proporcional de la muestra en algunas provincias, no se ha podido realizar un análisis segmentado a nivel provincial, por lo que tendremos que hacerlo a nivel autonómico, con las consiguientes limitaciones que ello conlleva en un análisis de la xenofobia como se ha puntualizado más arriba.

5. Son muchas las definiciones que, desde la sociología, se han dado de la modernización. Véase, por ejemplo, S.N. Eisenstardt (1970), Ensayos sobre el cambio social y la modernización, Madrid, Tecnos; C. Solé (1976), Modernización: un análisis sociológico, Barcelona, Edicions 62; Ídem (1999), Modernidad y modernización, Barcelona, Anthropos, y T. Carnero Rabat (1992), Modernización, desarrollo político y cambio social, Madrid, Alianza. 
fin, la meta siempre inalcanzable, ya que no existe sociedad moderna completa o concluida.

Siete son los principios básicos, entre otros, que sustentan a la teoría de la modernización: en primer lugar, su unidad de análisis, establecida territorialmente en los estados, las regiones o las comunidades autónomas; en segundo lugar, la unidad de medida basada en los indicadores de desarrollo, como la renta per cápita, la alfabetización, etc.; en tercer lugar, la tendencia a la convergencia; en cuarto lugar, la unidireccionalidad al desarrollo; en quinto lugar, la disimilaridad histórica de los estados hacia el desarrollo; en sexto lugar, la dialéctica y la confrontación con la tradición, y, por último, la validez e interiorización de los valores modernos.

No obstante, el proceso propio de la difusión de la modernización (desarrollo económico, social, cultural y político) dentro de un mismo estado facilita la aparición de diferencias en cuanto a la implantación de dicha modernización entre las diferentes nacionalidades o regiones.

Si partimos de los hechos diferenciales entre Andalucía y Cataluña en la mayoría de los indicadores socioeconómicos y políticos, el caso que nos ocupa, basado en los comportamientos y las actitudes hacia la xenofobia, no va a ser una excepción, sobre todo si, además, tenemos en cuenta tres hechos diferenciales importantes que entroncan con los principios teóricos y empíricos que afectan más determinantemente al racismo y a la xenofobia.

Los hechos diferenciales son, entre otros, los siguientes: primero, mientras tradicionalmente Andalucía ha sido una sociedad de emigración, Cataluña se ha significado por ser de inmigración. En segundo lugar, mientras en Cataluña el nacionalismo tiene una presencia importante, tanto en el ámbito político como económico y sociocultural, puesto que es, con el País Vasco y Galicia, la comunidad que ha mantenido una mayor conciencia nacionalista, Andalucía ha carecido de dicha conciencia en cualquier momento histórico en el que nos situemos, por lo que es residual la presencia del nacionalismo en dicha comunidad. Por último, los niveles de educación son más elevados en Cataluña que en Andalucía ${ }^{6}$, y es una constante que, a mayor nivel educativo, menor es el nivel de xenofobia de una sociedad o que diluye su efecto en una medida más elevada, como tendremos ocasión de comprobar más adelante.

En definitiva, nuestro punto de partida y también el de llegada es describir y analizar por qué la modernidad se entiende como un disolvente de las opiniones y de las actitudes ante la xenofobia, de la misma forma que, entre otros efectos, la modernidad mediatiza sobre las opiniones y las actitudes ante la religión impregnándola de efectos secularizadores.

Por otra parte, el concepto de densidad puede abordarse desde dos perspectivas: una de carácter demográfico, que responde a la necesidad de medir el

6. Véase, a este respecto, R. Gobernado Arribas (1996), «Niveles educativos y estratificación social», en R. Gobernado Arribas (coord.), Análisis comparado de las estructuras sociales de Andalucía y Cataluña, Málaga, Universidad de Málaga y Universidad de Almería , 77-110. 
número de inmigrantes y su distribución y concentración en el espacio, y otra de carácter sociológico, mayoritaria en los estudios sobre los efectos de la inmigración, que intenta analizar el número de inmigrantes en una población determinada atendiendo a su lugar de procedencia y a su proceso de incorporación segmentada. Este hecho cuantitativo tiene consecuencias cualitativas importantes, al menos en dos ámbitos: en el que afecta a la estructura del Estado del bienestar y en el relacional, especificado en el modelo de convivencia entre los diferentes grupos que interactúan en España.

Además, hemos creído conveniente fundamentar nuestra investigación en el índice de xenofobia a partir de modelos previamente construidos y contrastados empíricamente. Así, hemos elegido el índice elaborado por Díez Nicolás en sus obras Actitudes hacia los inmigrantes y Las dos caras de la inmigración ${ }^{7}$.

A partir de la elaboración del índice de xenofobia y su comparación en las dos comunidades autónomas analizadas, se ha llevado a cabo un análisis de regresión múltiple para observar las diferentes variables que parecen explicar mejor el citado índice. A tal efecto, se han creado tres modelos de regresión: el primero, a partir de las variables sociodemográficas y socioeconómicas más significativas; el segundo se fundamenta en una serie de variables sociopolíticas y culturales, y el tercero, relacionado con la inmigración, ha intentado explicar la xenofobia a partir del sentimiento de los entrevistados hacia los gitanos y diferentes grupos de inmigrantes, con el fin de precisar cuales de éstos son los mejores predictores de la xenofobia.

\section{Sobre el concepto de xenofobia y racismo}

Antes de introducirnos en los datos, conviene puntualizar lo que entendemos por xenofobia y su diferencia con otro concepto que, en muchas ocasiones, se

7. J. Diez Nicolás (1998), Actitudes hacia los inmigrantes, Madrid, Ministerio de Trabajo y Asuntos Sociales, Dirección General del Instituto de Migraciones y Servicios Sociales; Ídem (2005), Las dos caras de la inmigración, Madrid, Ministerio de Trabajo y Asuntos Sociales. Creemos que la validez demostrada por los datos procedentes del CIRES y posteriormente de ASEP a lo largo de la década de 1990 y hasta el año 2007, en multitud de investigaciones de todo tipo, es un motivo suficientemente objetivo para apuntalar dicha decisión. Además, se ha creído conveniente construir un índice de xenofobia y no de racismo, aparte de por el interés comparativo antes señalado, por dos motivos, aún siendo conscientes de la difícil delimitación entre los dos conceptos, como podrá comprobarse en el epígrafe siguiente: el primero, como se verá a continuación, basado en la secuencia definitoria de ambos conceptos; mientras la xenofobia es una actitud de rechazo o exclusión de tipo defensivo, por desconocimiento y miedo hacia los extranjeros, el racismo centra dicha actitud negativa especialmente en argumentos raciales; el segundo, de tipo metodológico, donde el concepto de actitud se entiende como respuestas verbales o simbólicas de aceptación o rechazo, idea que engloba también a las opiniones, entendidas éstas como expresiones verbales de creencias, actitudes y valores. En este sentido, las preguntas incluidas en la construcción del índice de xenofobia (véase nota 14) tienen un carácter más de opinión que de constatación de comportamientos racistas. 
ha utilizado como su sinónimo: el racismo. Como ya se ha definido anteriormente ${ }^{8}$, entendemos por xenofobia el comportamiento o la actitud que desarrolla un grupo social o étnico (en este caso, etnofobia) frente al temor o la prevención respecto de otros grupos (étnicos, sociales o nacionales) a los que se les considera extranjeros. Etimológicamente, procede de dos palabras griegas: xeno, que significa 'extranjero' o 'huésped', y fobia, que se entiende por 'miedo a', o 'prevenir contra'. Por otra parte, cuando hablamos de xenofobia, indirectamente también lo asociamos al concepto de racismo. Así, entendemos por racismo una ideología que promueve comportamientos o actitudes de odio y rechazo a personas que tienen características físicas diferentes a las del propio grupo étnico o racial.

Así definidos, ambos conceptos no pueden entenderse de la misma forma: mientras el racismo es una ideología y, por tanto, ésta debe articularse a través de organizaciones políticas y movimientos sociales que la sustenten y la apoyen, la xenofobia es un comportamiento de miedo hacia los extranjeros y se muestra en expresiones o acciones, en muchas ocasiones espontáneas, hacia o contra individuos pertenecientes a grupos étnico-culturales desconocidos que producen desconcierto y temor en el propio ${ }^{9}$. A estas diferencias, habría que añadir un denominador común paradigmático: la intolerancia.

No obstante, entre esta diferenciación extrema, el espacio del racismo cuenta con posiciones intermedias que Wieviorka concreta en tres: la primera, en torno a las relaciones de dominación existentes en la estructura social, cuyo caso paradigmático son las relaciones raciales en los Estados Unidos; la segunda, la acción histórica especificada en los proyectos de exclusión y destrucción hacia colectivos raciales concretos, como en el antisemitismo de los años treinta; la tercera, la acción social llevada a cabo por movimientos sociales o comunitarios de tipo nacionalista o religioso. En los casos extremos, estos tres ejes del racismo «son susceptibles de desembocar en proyectos políticos» ${ }^{10}$. En este ámbito de diferenciación conceptual entre el racismo y la xenofobia, otros

8. G. Herranz de Rafael (2002), « La xenofobia en Andalucía y Cataluña: un análisis comparativo", en La sociedad: Teoría e investigación, Madrid, CIS, 175-176; Ídem, "Xenofobia: un estudio comparativo en barrios y municipios almerienses», op. cit.

9. Por otra parte, los procesos de expulsión, refugio y deportación están muy relacionados con la xenofobia. Como dice Hidalgo, «tanto antes como ahora, la expulsión de un grupo humano de su lugar de residencia (judíos o cristianos, hutus o kurdos, sioux o bosnios), bien por acuerdos políticos entre estados o por decretos políticos de un estado, reviste un claro sesgo xenófobo [...] Normalmente son minorías en litigio con mayorías que los consideran extraños, extranjeros, "distintos" y que, por alguna razón añadida, los odian, sienten una fobia o una aversión hacia ellos que les hace víctimas de ese sacrificio simbólico que es el destierro» (A. Hidalgo Tuñón (1996), «Xenofobia», en F.J. Blázquez Ruiz (dir.), 10 palabras clave sobre racismo y xenofobia, Navarra, Verbo Divino, 132. Un ejemplo actual lo tenemos en Sudáfrica, donde más de 10.000 mozambiqueños han tenido que abandonar el país como consecuencia de los brotes xenófobos provocados por sudafricanos que acusan a los africanos «de ocupar sus empleos y alimentar una ola de delitos violentos» (El Mundo, 23 de mayo de 2008, 39).

10. M. Wieviorka (1992), El espacio del racismo, Barcelona, Paidós, 180. 
autores han visto más similitudes que diferencias ${ }^{11}$, lo cual constata la extrema dificultad delimitativa de ambos conceptos.

Por otra parte, las acciones o actitudes xenófobas pueden ser de diferente índole y pueden venir motivadas bien por elementos culturales intergrupales, como los prejuicios o los estereotipos aprendidos e interiorizados de otras culturas o pueblos, bien por connotaciones políticas más acentuadas, por ejemplo: utilizándolos como chivos expiatorios en momentos de crisis socioeconómicas o políticas, como fue, por ejemplo, el caso Dreifuss en Francia. Además, estarían otras situaciones que añadir aparte y como consecuencia de la mayor densidad de población extranjera, como son, entre otras, la posible competencia en la consecución de un puesto de trabajo; la vecindad con individuos pertenecientes a grupos etnoculturales no aceptados; la utilización de un mismo espacio lúdico o de ocio; el equilibrio en la integración de los niños en las escuelas; la aceptación de las diferentes manifestaciones culturales, como la utilización del velo por parte de mujeres musulmanas en instituciones públicas, etc. ${ }^{12}$.

\section{El concepto de densidad}

El concepto de densidad poblacional lo utilizó Durkheim en La división del trabajo social (1893) y Wirth en su artículo «El urbanismo como forma de vida» $(1938)^{13}$, pero, en nuestro caso, el concepto de densidad poblacional y el de densidad moral en sentido durkheimiano van a diferir. Durkheim establece la idea de que la densidad demográfica y moral están relacionadas con la división del trabajo, lo cual produce un mayor contacto de unos individuos con otros y, por tanto, existe más dependencia mutua y solidaridad y cooperación entre ellos. Sin embargo, la evidencia inmigratoria, al introducir la variable interviniente etnocultural, viene a establecer todo lo contrario: a mayor densidad poblacional, menor densidad moral en sentido durkheimiano.

Esta idea se explica si partimos del precedente axiológico durkheimiano que establece que, en primer lugar, debe darse una sociedad cuyos miembros

11. Véase, a este respecto, P.A. Taguieff (1988), La force du préjugé: Essai sur le racisme et ses doubles, París, Le Découverte; T.A. van Dijk (2003), Ideologia y discurso, Barcelona, Ariel; M. Banton (1983), Racial and ethnic competition, Cambridge, Cambridge University Press; Hechter (1986), "Rational choice theory and the study of race and ethnic relations», en J. Rex y D. Mason (eds.), Theory of race and ethnic relations, Cambridge, Cambridge University Press, 264-279; M. Barker, The new racism: conservatives and the ideology of the tribe, Londres, Junction Book; E. Balibar y I. Wallerstein (1991), Raza, clase y nación, IEPALA, y C. Solé (1991), Trabajadores extranjeros en Cataluña. ¿Integración o racismo?, Madrid, CIS, 22.

12. Véanse, entre otros, D. Macedo (2006), The globalization of racism, Boulder, Paradigm Publisher; F. Nuamjoh (2006), Insiders and outsiders: citizenship and xenophofia in contemporary southern Africa, Nueva York, Palgrave, y E. Cashdan (2001), "Ethocentrism and xenophobia a cross cultural study», Current Antropology, 42 (5), 760-765.

13. L. Wirth (1962), El urbanismo como forma de vida, Buenos Aires, Ediciones 3, 1962. 
estén unidos por unos valores comunes; en segundo lugar, que entre esos valores se encuentre que esa sociedad exista y persista, y, en tercer lugar, debe estar presente la voluntad de los miembros de esa sociedad para continuar viviendo juntos. A partir de esta situación axiológica, se produce un aumento de la densidad moral, situación que no se cumple en la mayoría de las sociedades que están pasando por un proceso de inmigración y, en mayor medida, en aquellos lugares donde se produce más densidad.

\section{Hipótesis y datos}

Partimos de dos hipótesis, que, aunque diferentes, pueden entenderse como complementarias. En primer lugar, y respecto a la densidad (volumen de inmigrantes que residen en un determinado hábitat en proporción a su número de habitantes), se espera que la xenofobia, en términos de conducta y actitudes, aumente en un determinado hábitat cuanto mayor sea el número de inmigrantes ${ }^{14}$ y cuanto menor sea, proporcionalmente, el número de habitantes autóctonos.

La densidad, junto con otros factores intervinientes ${ }^{15}$, como el crecimiento exponencial de inmigrantes en un corto periodo de tiempo que hace insuficiente el periodo de interiorización y asimilación de las nuevas condiciones de convivencia interétnicas, posibilitan en mayor medida las conductas y las actitudes xenófobas. Su alcance, no obstante, diferirá de unos grupos étnico-culturales a otros, como veremos más adelante.

En segundo lugar, se entiende que la modernización es un disolvente frente a la xenofobia, por lo que, cuanto más moderna sea una sociedad, menor índice de xenofobia mostrará.

Con respecto a los datos, se han utilizado las encuestas que CIRES y ASEP han realizado sobre la inmigración de los años 1991 a 2007, ambos incluidos. No obstante, tanto el índice de xenofobia como los modelos de regresión se han realizado a partir de una muestra agregada del año 2002 al 2007.

14. A esta misma conclusión llegan otras investigaciones, aún aplicando otras técnicas multivariantes, como la realizada por Mari Ángeles Cea, quien, a partir de la creación de una tipología de acuerdo con diferentes actitudes frente a la inmigración (tolerantes, ambivalentes y reacios), llega a la conclusión que los reacios han aumentado, en la misma proporción que los ambivalentes, a medida que ha ido aumentando el volumen de inmigrantes (M.A. Cea (2004), La activación de la xenofobia en España. ¿Qué miden las encuestas?, Madrid, CIS. También ver otra tipología interesante para el estudio de la xenofobia, entre utilitaristas, diferencialistas y pluralistas, en M. Pérez Yruela y T. Desrues (2005), Opinión de los españoles en materia de racismo y xenofobia, Madrid, Ministerio de Trabajo y Asuntos Sociales, Observatorio Español del Racismo y la Xenofobia, 108.

15. Mari Ángeles Cea incluye, además de la intensificación de la presencia de inmigrantes, que "puede acrecentar los sentimientos de alarma en la población autóctona, surgiendo en ésta percepciones de "avalancha" o estados psicóticos de "invasión"”, el miedo a la pérdida de la identidad nacional y cultural, así como la disminución de los logros socioeconómicos y jurídicos conseguidos. «Todo ello puede propiciar la activación de la xenofobia, hasta entonces aletargada o meramente episódica» (ibídem, «Introducción», IX). 
Por último, para el cálculo de índices, se tuvieron en cuenta los siguientes: xenofobia, materialismo y postmaterialismo, posición social, estatus socioeconómico familiar y políticas en extranjería del Estado ${ }^{16}$.

\section{Análisis de los resultados}

\subsection{Un estudio descriptivo de la xenofobia}

Como puede observarse en la tabla, la evolución del índice de xenofobia en Andalucía y Cataluña de 1991 a 2007 ha sido bajo y constante en ambas comunidades, con una ligera tendencia a incrementarse en los últimos años. No obstante, las medias de xenofobia son más acentuadas en Andalucía que en Cataluña: para el año 2007, la diferencia es de 1,37 puntos. Si lo comparamos con los datos nacionales, eligiendo los dos años con mayor índice de xenofobia (1992: 2,9 y 2002: 2,7), observamos que Andalucía se encuentra, en ambos casos, por encima de la media española en casi un punto para el primer año y de 0,73 puntos para el segundo, mientras Cataluña lo está por debajo, en 0,78 y 0,72 puntos respectivamente. En definitiva, este incremento del

16. 1. Índice de xenofobia. Las preguntas utilizadas para elaborar este índice de xenofobia han sido las actitudes básicas hacia la inmigración. Se han medido a través de cinco frases de acuerdo o desacuerdo que se propusieron a los entrevistados: a) Solo se debería admitir a trabajadores de otros países cuando no haya españoles para cubrir esos puestos de trabajo. b) Bastante difícil es la situación económica de los españoles como para, además, tener que destinar dinero a ayudar a los inmigrantes. c) Se diga lo que se diga, a todos nos molestaría que nuestros hijos tuvieran compañeros de otras razas en sus escuelas. d) La inmigración extranjera acabará provocando que España pierda su identidad. $e$ ) Los ciudadanos de cualquier país deberían tener derecho a establecerse en cualquier otro país, sin ningún tipo de limitaciones. A quienes respondieron estar «muy de acuerdo» $\mathrm{o}$ "de acuerdo» con las frases $a), b), c)$ y $d$ ), o «muy en desacuerdo» o «en desacuerdo» con la frase $e$ ), se les asignó un punto. Del mismo modo, se asignó un punto a los entrevistados que prohibirían a su hija tener relaciones con un hombre de cada uno de los siguientes grupos sociales: gitanos, norteafricanos, africanos de raza negra, sudamericanos y europeos del Este. Finalmente, se asignó también un punto a quienes juzgaron molestar la convivencia en el vecindario con sudamericanos, gitanos, africanos de raza negra y marroquíes.

2. Índice de materialismo o postmaterialismo. Este índice varía de 1 a 3, donde el 1 significa valores materialistas; el 3, valores postmaterialistas, y el 2, la opción mixta.

3. Índice de posición social. Es un índice sintético que combina ocho variables diferentes y dicotomizadas: sexo, edad, nivel educativo, estatus ocupacional, nivel de ingresos familiares mensuales, sector económico de actividad, tamaño del hábitat de residencia y centralidad geográfica.

4. Índice de estatus socioeconómico familiar. Basado en los ingresos familiares, la ocupación del cabeza de familia y el equipamiento del hogar. Combinando estos tres indicadores, se han definido cuatro niveles: alto, medio, medio-bajo y bajo.

5. Índice de políticas en extranjería del Estado. Construido con una escala de 0 a 6 puntos a partir de las respuestas afirmativas a la siguiente pregunta: «¿Cree que el Estado español debería proporcionar las siguientes ayudas a inmigrantes: acceso la vivienda, subsidio de paro, asistencia sanitaria, educación gratuita para los hijos, educación gratuita para su cónyuge, subsidio de paro para los emigrantes, cursos de español y cursos de formación profesional para inmigrantes?». 
Tabla 4. Evolución del índice de xenofobia en Andalucía

\begin{tabular}{lccc}
\hline Número de estudio & Media & N & Desviación típica \\
\hline $1991-03$ & 2,54 & 203 & 2,169 \\
$1992-03$ & 3,85 & 199 & 2,493 \\
$1993-03$ & 2,34 & 204 & 1,995 \\
$1994-03$ & 2,49 & 200 & 2,439 \\
$1995-03$ & 2,41 & 217 & 2,390 \\
$1995-10$ & 2,22 & 213 & 1,982 \\
$1996-12$ & 2,49 & 206 & 2,190 \\
$1997-12$ & 2,08 & 208 & 2,160 \\
$1998-11$ & 1,75 & 207 & 1,984 \\
$1999-10$ & 2,39 & 206 & 2,140 \\
$2000-10$ & 2,47 & 211 & 2,466 \\
$2001-11$ & 2,04 & 213 & 2,486 \\
$2002-09$ & 3,43 & 208 & 2,814 \\
$2003-09$ & 3,55 & 213 & 2,967 \\
$2004-11$ & 2,82 & 211 & 2,458 \\
$2005-11$ & 3,14 & 210 & 2,833 \\
$2006-09$ & 3,54 & 206 & 2,822 \\
$2007-10$ & 3,88 & 210 & 3,005 \\
\hline Total & 2,74 & 3745 & 2,532 \\
\hline
\end{tabular}

Fuente: CIRES y ASEP. Elaboración propia.

Tabla 5. Evolución del índice de xenofobia en Cataluña

\begin{tabular}{lccc}
\hline Número de estudio & Media & N & Desviación típica \\
\hline $1991-03$ & 2,32 & 193 & 1,895 \\
$1992-03$ & 2,12 & 191 & 1,962 \\
$1993-03$ & 2,13 & 193 & 2,101 \\
$1994-03$ & 2,08 & 194 & 1,769 \\
$1995-03$ & 2,11 & 187 & 1,970 \\
$1995-10$ & 2,49 & 189 & 2,036 \\
$1996-12$ & 2,16 & 194 & 1,958 \\
$1997-12$ & 2,55 & 196 & 2,189 \\
$1998-11$ & 1,87 & 194 & 1,974 \\
$1999-10$ & 1,85 & 196 & 1,885 \\
$2000-10$ & 1,65 & 193 & 1,717 \\
$2001-11$ & 2,02 & 193 & 1,983 \\
$2002-09$ & 1,98 & 192 & 2,078 \\
$2003-09$ & 1,66 & 193 & 1,692 \\
$2004-11$ & 1,58 & 192 & 1,586 \\
$2005-11$ & 1,75 & 193 & 1,279 \\
$2006-09$ & 2,26 & 192 & 1,692 \\
$2007-10$ & 2,51 & 187 & 1,644 \\
\hline Total & 2,06 & 3462 & 1,887 \\
\hline
\end{tabular}

Fuente: CIRES y ASEP. Elaboración propia. 
índice de xenofobia es debido al aumento considerable del número de inmigrantes en la sociedad española en general, así como en Andalucía y Cataluña en particular.

Si consideramos el índice de xenofobia a partir de la muestra agregada de los últimos seis años (2002-2007), podemos observar que la xenofobia sigue siendo baja en ambas comunidades, aunque en Andalucía es más alta, con una diferencia de 1,44 puntos.

De acuerdo con estos datos, podemos concluir que, y con respecto a la primera hipótesis, la mayor densidad de inmigrantes no parece ser determinante en el nivel de xenofobia existente en una sociedad, ya que Cataluña, con más del doble (13,48\%) de inmigrantes en el año 2007 que Andalucía, tiene un índice de xenofobia menor. Esto puede ser explicado también por las limitaciones metodológicas antes apuntadas, en el sentido de que las encuestas a muestras totales de la población reflejan opiniones y actitudes ante la inmigración poco reales, al asignarle el mismo peso a personas que viven en zonas con más de un $16 \%$ de extranjeros, como es el caso de Almería o Girona, que a otras donde no llega al 3\%, como son Sevilla, Córdoba o Jaén. Además, la mayoría de las citadas encuestas realizadas a la población española reflejan valoraciones positivas hacia la inmigración, dato que no se corresponde con el obtenido mediante otros estudios, en los que se ha tenido en cuenta una mayor segmentación de la población en función de la densidad de inmigrantes. Por ejemplo, en Almería, los extranjeros no comunitarios suponían el 8,95\% de la población almeriense a principios del año $2004^{17}$. Un total de 13 municipios de los 103 existentes en Almería superaban esta tasa, aunque había seis que destacaban sobremanera por rebasar el $14 \%$ y conformar un grupo singular. El índice de xenofobia alcanzó los 5,3 puntos para el año 2005, es decir, 2,16 puntos más que Andalucía y 3,55 más que Cataluña.

Respecto a la segunda hipótesis, se confirma en el sentido que, a mayor grado de modernización, menor es el índice de xenofobia. Andalucía y Cataluña son dos sociedades que están en diferentes estadios de modernización, pero Cataluña se encuentra en un nivel más avanzado que Andalucía, hecho que corrobora que el índice de xenofobia sea significativamente menor en Cataluña que en Andalucía, así como que esté por debajo de la media española.

Por otra parte, y de modo comparativo, si para el caso Andaluz el 43,4\% es nada o poco xenófobo, para el caso catalán representa algo más de dos tercios $(68,3 \%)$. En sentido contrario, los algo o muy xenófobos eran en Andalucía más de la mitad de la población (56,6\%), mientras que en Cataluña no llegaban a un tercio $(31,7 \%)$. Además, debe tenerse en cuenta que sólo algo más de una sexta parte $(14,4 \%)$ de la población andaluza puede considerarse muy xenófoba, mientras que en la catalana no llega al $2 \%$.

17. El porcentaje de población extranjera extracomunitaria (UE 25) en los municipios almerienses era la siguiente: La Mojonera (22,6\%), Pulpí (21,0\%), El Ejido (19,4\%), Níjar $(17,35 \%)$, Roquetas de Mar (16,6\%), Vicar (14,1\%). Globalmente, en la provincia de Almería: 9,0\%. 
Tabla 6. Índice de xenofobia en Andalucía (2002-2007, agregados)

\begin{tabular}{lcc}
\hline & Frecuencia & $\%$ \\
\hline 0 Nada xenófobo & 189 & 15,0 \\
1 & 165 & 13,1 \\
2 & 192 & 15,3 \\
3 & 212 & 16,9 \\
4 & 173 & 13,8 \\
5 & 86 & 6,8 \\
6 & 59 & 4,7 \\
7 & 45 & 3,6 \\
8 & 45 & 3,6 \\
9 & 42 & 3,3 \\
10 & 22 & 1,7 \\
11 & 9 & 0,7 \\
12 & 13 & 1,0 \\
13 & 4 & 0,3 \\
14 Muy xenófobo & 2 & 0,2 \\
\hline Total & 1.258 & 100 \\
Media de xenofobia & & 3,39 \\
\hline Desviación típica & & 2,84 \\
\hline
\end{tabular}

Fuente: ASEP. Elaboración propia.

Tabla 7. Índice de xenofobia en Cataluña (2002-2007, agregados)

\begin{tabular}{lcc}
\hline & Frecuencia & $\%$ \\
\hline 0 Nada xenófobo & 221 & 19,2 \\
1 & 331 & 28,8 \\
2 & 233 & 20,3 \\
3 & 185 & 16,1 \\
4 & 82 & 7,1 \\
5 & 59 & 5,1 \\
6 & 19 & 1,7 \\
7 & 10 & 0,9 \\
8 & 3 & 0,3 \\
9 & 2 & 0,2 \\
10 & 2 & 0,2 \\
11 & 2 & 0,2 \\
12 & 0 & 0 \\
13 & 0 & 0 \\
14 Muy xenófobo & 0 & 0 \\
\hline Total & 1.149 & 100 \\
Media de xenofobia & & 1,95 \\
Desviación tí́pica & & 1,71 \\
\hline
\end{tabular}

Fuente: ASEP. Elaboración propia. 
Tabla 8. Perfil de los entrevistados según el índice de xenofobia en Andalucía (2002-2007, agregado)

\begin{tabular}{|c|c|c|c|c|c|c|c|c|c|c|}
\hline & \multicolumn{2}{|c|}{ Nada xenófobo } & \multicolumn{2}{|c|}{ Poco xenófobo } & \multicolumn{2}{|c|}{ Algo xenófobo } & \multicolumn{2}{|c|}{ Muy xenófobo } & \multicolumn{2}{|c|}{ Total } \\
\hline & And. & Cat. & And. & Cat. & And. & Cat. & And. & Cat. & And. & Cat. \\
\hline \multirow[t]{2}{*}{ Total } & (189) & (189) & $(357)$ & (357) & (530) & (530) & (182) & (182) & (1.258) & $(1,25$ \\
\hline & $15,0 \%$ & $19,2 \%$ & $28,4 \%$ & $49,1 \%$ & $42,2 \%$ & $30,0 \%$ & $14,4 \%$ & $1,7 \%$ & & \\
\hline \multicolumn{11}{|l|}{ Sexo } \\
\hline arones & $48,1 \%$ & $54,3 \%$ & $47,9 \%$ & $48,9 \%$ & $48,9 \%$ & $42,0 \%$ & $52,2 \%$ & $26,3 \%$ & $49,0 \%$ & $47,5 \%$ \\
\hline Mujeres & $51,9 \%$ & $45,7 \%$ & $52,1 \%$ & $51,1 \%$ & $51,1 \%$ & $58,0 \%$ & $47,8 \%$ & $73,7 \%$ & $51,0 \%$ & $52,2 \%$ \\
\hline \multicolumn{11}{|l|}{ Edad } \\
\hline 30 año & $30,7 \%$ & $33,0 \%$ & $28,0 \%$ & $26,6 \%$ & $29,2 \%$ & $20,9 \%$ & $22,5 \%$ & $15,8 \%$ & $28,1 \%$ & $25,9 \%$ \\
\hline os & $22,2 \%$ & $17,6 \%$ & $21,3 \%$ & $19,3 \%$ & $20,2 \%$ & $17,7 \%$ & $14,8 \%$ & 5,3 & $20,0 \%$ & $18,3 \%$ \\
\hline os & $15,9 \%$ & $13,6 \%$ & $17,6 \%$ & $18,1 \%$ & $15,8 \%$ & $13,3 \%$ & $12,1 \%$ & $15,8 \%$ & $15,8 \%$ & $15,8 \%$ \\
\hline & $8,5 \%$ & $14,5 \%$ & $11,5 \%$ & $13,1 \%$ & $11,9 \%$ & $13,3 \%$ & $14,8 \%$ & - & $11,7 \%$ & $13,2 \%$ \\
\hline Más de 60 años & $22,8 \%$ & $21,3 \%$ & $21,6 \%$ & $22,9 \%$ & $22,8 \%$ & $34,8 \%$ & $35,7 \%$ & $63,2 \%$ & $24,3 \%$ & $26,8 \%$ \\
\hline \multicolumn{11}{|c|}{ Educación del entrevistado } \\
\hline Bin & $32,6 \%$ & $17,7 \%$ & $4,2 \%$ & $19,3 \%$ & $41,9 \%$ & $28,5 \%$ & $55,6 \%$ & 47,4 & $40,3 \%$ & $22,2 \%$ \\
\hline dia & $52,9 \%$ & $62,7 \%$ & $53,7 \%$ & $63,3 \%$ & $50,1 \%$ & $62,8 \%$ & $42,2 \%$ & 47,4 & $50,4 \%$ & $62,9 \%$ \\
\hline Alta & $14,4 \%$ & $19,5 \%$ & $12,1 \%$ & $17,0 \%$ & $8,0 \%$ & $8,7 \%$ & $2,2 \%$ & 5,3 & $9,3 \%$ & $4,8 \%$ \\
\hline \multicolumn{11}{|l|}{ Hábitat } \\
\hline & $2 \%$ & $\%$ & $3,0 \%$ & $12,8 \%$ & $24,0 \%$ & $24,3 \%$ & $14,8 \%$ & 6 & $23,5 \%$ & $17,2 \%$ \\
\hline no &, $9 \%$ & $50,7 \%$ & $54,1 \%$ & $59,0 \%$ & $50,9 \%$ & $58,3 \%$ & $50,5 \%$ & 52 & $4,8 \%$ & $57,1 \%$ \\
\hline Metropolitano & $6,9 \%$ & $33,5 \%$ & $17,9 \%$ & $28,2 \%$ & $25,1 \%$ & $17,4 \%$ & $34,6 \%$ & 10,5 & $21,7 \%$ & $25,7 \%$ \\
\hline \multicolumn{11}{|c|}{ Clase social subjetiva } \\
\hline Baia & $2,7 \%$ & 0 & $2,0 \%$ & $1,6 \%$ & $1,7 \%$ & $4,4 \%$ & $4,5 \%$ & & $2,4 \%$ & $2,8 \%$ \\
\hline Ila & $7 \%$ & $94,5 \%$ & $92,5 \%$ & $96,6 \%$ & $94,8 \%$ & $93,8 \%$ & $92,6 \%$ & 84,3 & $4,0 \%$ & $95,2 \%$ \\
\hline Alta & $1,6 \%$ & $2,8 \%$ & $5,5 \%$ & $1,8 \%$ & $3,4 \%$ & $1,8 \%$ & $2,8 \%$ & 5,3 & $3,7 \%$ & $2,0 \%$ \\
\hline \multicolumn{11}{|l|}{ Posición social } \\
\hline . & $46,6 \%$ &, $9 \%$ &, $8 \%$ & $24,6 \%$ & $39,8 \%$ & $35,9 \%$ & $41,2 \%$ & 2 & $40,5 \%$ & $27,9 \%$ \\
\hline & $45,5 \%$ & $3 \%$ & $8,7 \%$ & $58,3 \%$ & $49,4 \%$ & $54,2 \%$ & $51,6 \%$ & & $9,0 \%$ & $56,7 \%$ \\
\hline Alta & $7,9 \%$ & $20,8 \%$ & $13,4 \%$ & $17,0 \%$ & $10,8 \%$ & $9,9 \%$ & $7,1 \%$ & $5,3 \%$ & $0,6 \%$ & $15,4 \%$ \\
\hline \multicolumn{11}{|l|}{ Ideología } \\
\hline & $9 \%$ & $86,9 \%$ & $5,3 \%$ & $72,1 \%$ & $64,4 \%$ & $53,4 \%$ & $57,1 \%$ & $58,3 \%$ & $64,9 \%$ & $69,8 \%$ \\
\hline & $15,7 \%$ & $10,5 \%$ & $14,9 \%$ & $25,3 \%$ & $21,5 \%$ & $36,4 \%$ & $27,1 \%$ & 33,3 & $19,9 \%$ & $25,5 \%$ \\
\hline Derecha & $8,3 \%$ & $2,6 \%$ & $19,8 \%$ & $2,6 \%$ & $14,1 \%$ & $10,2 \%$ & $15,7 \%$ & $8,3 \%$ & $5,2 \%$ & $4,7 \%$ \\
\hline \multicolumn{11}{|c|}{ Sentimiento nacionalista } \\
\hline 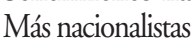 & & 3 & $10,1 \%$ & & $9,2 \%$ & $33,1 \%$ & 11 , & & & 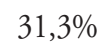 \\
\hline 8 & $4 \%$ & $\%$ & $65,8 \%$ & $0 \%$ & $74,0 \%$ & $45,6 \%$ & $78,0 \%$ & $21,1 \%$ & $1,5 \%$ & $52,6 \%$ \\
\hline Más españoles & $12,1 \%$ & $17,5 \%$ & $24,1 \%$ & $11,9 \%$ & $16,8 \%$ & $21,3 \%$ & $11,0 \%$ & 31,60 & $17,3 \%$ & $16,1 \%$ \\
\hline \multicolumn{11}{|c|}{ Postmaterialismo } \\
\hline 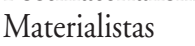 & $66,1 \%$ & $38,0 \%$ & 66,9 & $\%$ & 71 & $74,8 \%$ & $70,9 \%$ & 73 & $69,4 \%$ & $62,8 \%$ \\
\hline Postmaterialistas & s $33,9 \%$ & $62,0 \%$ & & & & 25 , & & & $5 \%$ & $7,2 \%$ \\
\hline \multicolumn{11}{|c|}{ Identificación espacial } \\
\hline Local & $81,0 \%$ & $45,2 \%$ & $80,7 \%$ & $42,9 \%$ & $78,8 \%$ & $49,0 \%$ & $80,6 \%$ & $52,6 \%$ & $79,9 \%$ & $45,3 \%$ \\
\hline Nacion & $12,3 \%$ & $31,8 \%$ & $15,6 \%$ & $50,4 \%$ & $19,9 \%$ & $46,6 \%$ & $19,4 \%$ & $42,1 \%$ & $17,5 \%$ & $45,6 \%$ \\
\hline Supranacional & $6,7 \%$ & $23,0 \%$ & $3,7 \%$ & $6,8 \%$ & $1,3 \%$ & $4,4 \%$ & - & $5,3 \%$ & $2,6 \%$ & $9,1 \%$ \\
\hline \multicolumn{11}{|c|}{ Viajes al extranjero } \\
\hline No & & & ניצמש & & & & & & & \\
\hline Sí & $2 \%$ & $84,2 \%$ & $57,7 \%$ & $4 \%$ & $43,2 \%$ & $71,0 \%$ & $26,9 \%$ & $42,1 \%$ & $47,2 \%$ & $78.7 \%$ \\
\hline
\end{tabular}

Fuente: ASEP. Elaboración propia. 
Tabla 9. Perfil de la xenofobia en Andalucía y Cataluña

\begin{tabular}{|c|c|c|c|}
\hline $\begin{array}{l}\text { Más xenófobo } \\
\text { en Andalucía }\end{array}$ & $\begin{array}{c}\text { Más xenófobos } \\
\text { en Catalunya }\end{array}$ & $\begin{array}{l}\text { Menos xenófobos } \\
\text { en Andalucía }\end{array}$ & $\begin{array}{c}\text { Menos xenófobos } \\
\text { en Cataluña }\end{array}$ \\
\hline + edad. & + edad. & + jóvenes. & + jóvenes. \\
\hline Nivel educativo bajo. & $\begin{array}{l}\text { Nivel educativo } \\
\text { bajo. }\end{array}$ & $\begin{array}{l}\text { Nivel educativo } \\
\text { alto y medio. }\end{array}$ & $\begin{array}{l}\text { Nivel educativo } \\
\text { alto y medio. }\end{array}$ \\
\hline Rural y urbano. & Rural. & Metropolitano. & Metropolitano. \\
\hline Posición social media. & Posición social baja. & Posición social alta. & $\begin{array}{l}\text { Posición social alta } \\
\text { y media. }\end{array}$ \\
\hline De centro y de derechas. & De centro y de derechas. & De izquierdas. & De izquierdas. \\
\hline Identificación dual & $\begin{array}{l}\text { Nacionalistas } \\
\text { y españolistas. }\end{array}$ & $\begin{array}{l}\text { Más nacionalistas } \\
\text { y españolistas. }\end{array}$ & Identificación dual. \\
\hline Materialista. & Materialista. & Postmaterialista. & Postmaterialista. \\
\hline Local y nacional. & Local y nacional. & Supranacional. & Supranacional. \\
\hline No viaja al extranjero. & No viaja al extranjero. & Sí viaja al extranjero. & Sí viaja al extranjero. \\
\hline
\end{tabular}

Fuente: elaboración propia.

Comparando los datos a nivel de las dos comunidades autónomas, podemos concluir que, aunque en ambas comunidades hay un bajo nivel de xenofobia, con tendencia creciente, en Andalucía los porcentajes de los algo o muy xenófobos empiezan a ser preocupantes, puesto que alcanzan a más de la mitad de la población en el último sexenio.

Respecto al perfil socioeconómico de aquellos individuos andaluces y catalanes que pueden considerarse más o menos xenófobos, los datos evidencian una gran similitud, aunque con algunas diferencias.

Para el caso de ambas comunidades, el resumen de los más o menos xenófobos sería el que presentamos en la tabla.

Como se puede comprobar, las excepciones para el caso andaluz entre los más xenófobos se especifican en el hábitat, incluyendo tanto el rural como el urbano, la posición social media ${ }^{18}$ y que se identifican por igual tanto con su comunidad autónoma como con España. Para esta última variable, en Cataluña los más xenófobos son los que se identifican como nacionalistas. Respecto a los menos xenófobos, las variaciones se producen en Andalucía, en aquellos que se identifican como nacionalistas o españolistas, mientras que en Cataluña predominan los que tienen una identificación dual y tienen una posición social alta y media.

18. En el estudio antes citado sobre la xenofobia en Almería, los individuos con baja y media posición social también se adscribían entre los más xenófobos. De hecho, en el análisis de los modelos de regresión aplicados al estudio de los barrios almerienses con alta densidad de inmigrantes, la posición social tenía el signo positivo, es decir, a mayor posición social, mayor era su grado de xenofobia (op. cit., 125). Por otra parte, para el caso español, esta variable siempre tiene la misma tendencia, en el sentido que, a menor posición social, mayor grado de xenofobia y viceversa. 


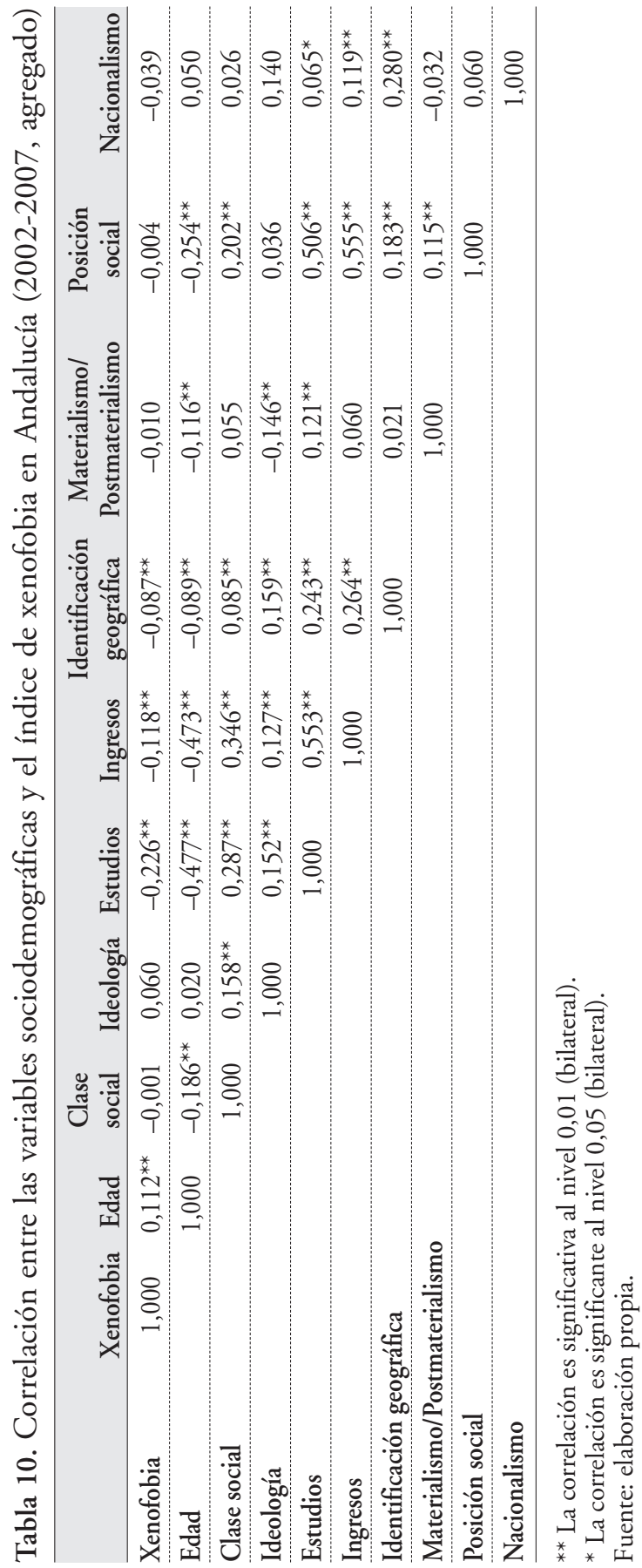




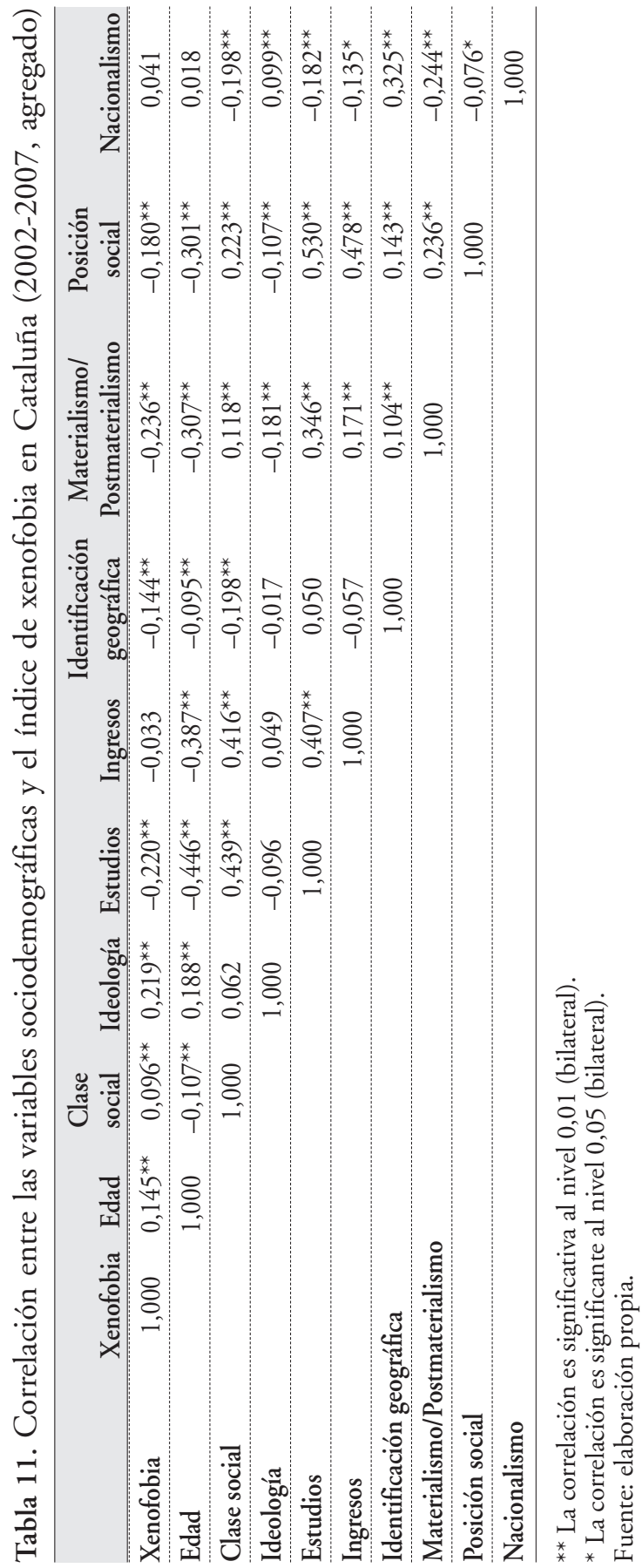


Tabla 12. Análisis de regresión de diversos indicadores sociodemográficos (variables independientes) sobre el índice de xenofobia (variable dependiente) en Andalucía y Cataluña (2002-2007, aregado)

\begin{tabular}{lcc}
\hline & Andalucía & Cataluña \\
\hline Coeficiente de correlación múltiple R & 0,389 & 0,392 \\
\hline Coeficientes de regresión estandarizados $\beta:$ & & \\
Nivel educativo & $-0,396$ & $-0,195$ \\
Ideología (derechismo) & 0,129 & 0,125 \\
Posición social & 0,198 & $-0,108$ \\
Postmaterialismo & $-0,058$ & $-0,177$ \\
Identificación espacial & $-0,039$ & 0,009 \\
Nacionalismo & $-0,146$ & $-0,018$ \\
Ingresos familiares & $-0,028$ & 0,181 \\
Edad & 0,019 & 0,028 \\
\hline
\end{tabular}

Fuente: ASEP. Elaboración propia.

\subsection{Un estudio explicativo de la xenofobia}

Avanzando algo más en la investigación, se llevó a cabo un análisis de regresión múltiple para observar las diferentes variables que parecen explicar mejor el índice de xenofobia. Este estudio se ha realizado en tres partes, mediante tres modelos de regresión múltiple, donde el índice de xenofobia aparece como variable dependiente y son las independientes diferentes para la mayor parte de los modelos.

En primer lugar, y antes de entrar en los modelos de regresión, nos interesaba saber el grado y el signo de asociación entre el índice de xenofobia y las variables sociodemográficas más significativas. Para el caso andaluz, el índice de xenofobia tiene un mayor nivel de asociación en relación con las variables sobre el nivel de estudios, los ingresos, la edad y la identificación geográfica. Para Cataluña, encontramos que la tiene con el materialismo y el postmaterialismo, los estudios, la ideología, la posición social, la edad y la identificación geográfica ${ }^{19}$.

Respecto del primer modelo, las variables independientes incluidas coinciden con las utilizadas para describir el perfil de los entrevistados según el índice de xenofobia.

El coeficiente de correlación fue del 0,38 para Andalucía y del 0,39 para Cataluña, por lo que las variables sociodemográficas y sociopolíticas

19. Siguiendo el criterio establecido al calcular el índice de xenofobia que fluctuaba de 0 a 14 puntos, donde los valores bajos señalaban menor grado de xenofobia y los altos, mayor grado de xenofobia, el resto de variables sociodemográficas seguirán el mismo esquema: los valores bajos corresponden a los grados más bajos de la variable, por ejemplo: bajo nivel educativo, etc. Para el resto de variables, el criterio fue el siguiente:

- Ideología: bajo hacia posiciones de izquierda y alto hacia la derecha.

- Identificación geográfica: bajo, identificación localista, y alto, universalista.

- Nacionalismo: bajo, sentimiento e identificación nacionalistas, y alto, más españolista.

- Postmaterialismo: bajo, posición más materialista, y alto, más postmaterialista. 
Tabla 13. Predictores más significaivos en Andalucía y Cataluña

\begin{tabular}{lcr}
\hline & Andalucía & Cataluña \\
\hline Nivel educativo & $(-0,396)$ & $(-0,195)$ \\
Posición social & $(0,198)$ & $(-0,108)$ \\
Nacionalismo & $(-0,146)$ & $(-0,177)$ \\
Ideología & $(0,129)$ & $(0,125)$ \\
Ingresos familiares & & $(0,181)$ \\
\hline
\end{tabular}

Fuente: elaboración propia.

tienen para ambos casos un aceptable poder explicativo en la variación en el grado de xenofobia. Además, los predictores más significativos, al tener los coeficientes de regresión estandarizados más altos, quedan reflejados en la tabla 13.

De acuerdo con estos datos, podemos decir que, en Andalucía y en Cataluña:

1. Cuanto mayor es el nivel educativo de los andaluces y los catalanes, menor es su grado de xenofobia.

2. En Andalucía, cuanto más alta es la posición social del sujeto, mayor es su grado de xenofobia, tendencia contraria de lo que ocurre en Cataluña, donde la menor posición social es la que genera más opiniones y conductas xenófobas.

3. En ambas comunidades, cuanto más a la izquierda se posicionan los individuos, menor es su grado de xenofobia, tendencia que se invierte a medida que se sitúan en disposiciones más derechistas.

4. La identificación nacionalista está en Andalucía en una posición negativa con la xenofobia, de manera que los más nacionalistas son los menos xenófobos.

5. En Cataluña, cuanto más se acercan los individuos a posiciones postmaterialistas, menor es el grado de xenofobia.

Tabla 14. Análisis de regresión de diferentes variables sociopolítico-culturales (variables independientes) sobre el indice de xenofobia (variable dependiente) en Andalucía y Cataluña (2002-2007), agregado)

\begin{tabular}{lcc}
\hline & Andalucía & Cataluña \\
\hline Coeficiente de correlación múltiple $\mathrm{R}=$ & 0,483 & 0,437 \\
\hline Coeficientes de regresión estandarizados $\beta:$ & & \\
Estado (políticas) & $-0,403$ & $-0,257$ \\
Nivel educativo & $-0,211$ & $-0,145$ \\
Postmaterialismo & $-0,043$ & $-0,179$ \\
Práctica religiosa & $-0,106$ & $-0,136$ \\
Ideología (radicalismo) & 0,002 & $-0,048$ \\
Nacionalismo (radicalismo) & $-0,156$ & 0,150 \\
Edad & $-0,003$ & $-0,028$ \\
\hline
\end{tabular}

Fuente: elaboración propia. 
6. Por último, y respecto a los ingresos familiares de los catalanes, se observa que, a mayores ingresos, mayor es la tendencia xenófoba.

En el segundo modelo, se pretendía observar el comportamiento del índice de xenofobia a partir de una serie de valores extremos de un grupo de tres variables de características exclusivamente sociopolítico-culturales, buscando la posible conexión entre el citado índice y esos valores sociopolítico-culturales extremos, además de mantener algunas variables ya incluidas en el modelo anterior y la agregación de una nueva: la práctica religiosa. Las tres variables independientes incluidas en el modelo de regresión fueron: políticas de estado, ideología (radicalismo) y nacionalismo (radicalismo) ${ }^{20}$. Como puede comprobarse, este modelo explica algo más que el anterior la xenofobia, al obtener un coeficiente de correlación múltiple más alto: de 0,48 para Andalucía y de 0,43 para Cataluña.

Respecto a las tres variables extremas, observamos que, a excepción de la ideología, las otras dos tienen un nivel explicativo aceptable. Para el caso de las políticas del Estado, al tener un signo negativo, informan de que tanto los andaluces como los catalanes, cuanto más a favor están de que éstas favorezcan al inmigrante, tanto en el acceso a una vivienda como a la asistencia sanitaria, etc., menor es su grado de xenofobia. El nacionalismo radical en Cataluña guarda relación directa con el nivel de xenofobia, pues a mayor identificación con planteamientos nacionalistas, incluyendo el españolista, mayores serán las conductas y las actitudes xenófobas frente a los extranjeros. En cambio, en Andalucía pasa lo contrario, los más nacionalistas tienden a ser menos xenófobos. Algo parecido ocurre en las dos comunidades con la práctica religiosa. En Andalucía, el signo negativo informa que cuanto mayor es la práctica religiosa, mayor es la tendencia xenófoba, mientras que en Cataluña ocurre todo lo contrario: los más practicantes muestran actitudes y conductas menos xenófobas.

En el tercer modelo se ha intentado explicar la xenofobia a partir del sentimiento de los entrevistados hacia los gitanos y diferentes grupos de inmigrantes, con el fin de precisar cuáles de éstos son los mejores predictores de la xenofobia.

Como puede apreciarse, el coeficiente de correlación fue del 0,45 para Andalucía y del 0,39 para Cataluña, por lo que los sentimientos hacia estos

20. Políticas de estado: construida con una escala de 0 a 6 puntos a partir de las respuestas afirmativas a la pregunta siguiente: «¿Cree que el Estado español debería proporcionar las siguientes ayudas a inmigrantes: acceso a la vivienda, subsidio de paro, asistencia sanitaria, educación gratuita para hijos, educación gratuita para el cónyuge, subsidio de paro para los emigrantes, cursos de español y cursos de formación profesional para inmigrantes?».

Ideología (radicalismo): variable construida a partir de la ideología política donde la opción centro toma el valor menor y la extrema derecha y la extrema izquierda, el valor mayor.

Nacionalismo (radicalismo): variable construida a partir del nacionalismo, donde la identificación dual, opción «tan andaluz como español», toma el valor menor, y la identificación españolista y nacionalista, es decir, «solo español» y «solo andaluz», el valor mayor. 
Tabla 15. Análisis de regresión del sentimiento hacia los gitanos y diferentes grupos de inmigrantes (variables independientes) sobre el indice de xenofobia (variable dependiente) en Andalucía y Cataluña (2002-2007), agregado)

\begin{tabular}{lcc}
\hline & Andalucía & Cataluña \\
\hline Coeficiente de correlación múltiple $\mathrm{R}=$ & 0,459 & 0,398 \\
\hline Coeficientes de regresión estandarizados $\beta:$ & & \\
Sudamericanos & $-0,114$ & $-0,065$ \\
Gitanos & $-0,092$ & $-0,126$ \\
Árabes y musulmanes & $-0,187$ & $-0,277$ \\
Judíos & 0,034 & 0,011 \\
Africanos negros & $-0,175$ & $-0,007$ \\
Edad & $-0,003$ & $-0,028$ \\
\hline
\end{tabular}

Fuente: elaboración propia.

colectivos de inmigrantes tienen para ambos casos un aceptable poder explicativo en la variación en el grado de xenofobia.

El análisis de los coeficientes de regresión permite observar dos realidades diferentes en las comunidades analizadas respecto a los sentimientos hacia los diversos colectivos de inmigrantes: por una parte, tanto en Andalucía como en Cataluña, los sentimientos hacia los árabes y musulmanes son los que contribuyen más a explicar la xenofobia, en el sentido de que, cuanto más baja es la evaluación de ambos grupos sociales, mayor es el grado de xenofobia de los individuos. En efecto, este mayor rechazo a este grupo puede venir motivado por dos hechos: en primer lugar, a su mayor presencia tanto en Andalucía como en Cataluña. Si analizamos los datos, vemos que es el segundo colectivo más numeroso en Andalucía, si exceptuamos los europeos comunitarios, y el segundo en Cataluña, por encima de éstos últimos, y, en segundo lugar, como señalan la mayoría de los estudios sobre inmigración, por la percepción que tienen los autóctonos a su rechazo a integrarse en la sociedad española. Por otra parte, mientras en Cataluña los sentimientos de rechazo se focalizan hacia el colectivo de gitanos ${ }^{21}$, como segundo grupo de importancia, en Andalucía se concreta en los africanos negros y los sudamericanos. Debe aclararse, no obstante, que la evaluación de gitanos, sudamericanos y africanos negros en ambas comunidades también está inversamente correlacionada con el índice de xenofobia, pero su contribución a la explicación de la xenofobia añade algo menos a la explicación de la xenofobia que proporcionan la evaluación de los árabes y los musulmanes. Por último, solamente el colectivo de judíos adquiere un sentimiento positivo en las dos comunidades analizadas.

21. En la década de 1990, a nivel nacional, el colectivo de gitanos y, en menor medida, los africanos negros, eran los que establecían un nivel más elevado de rechazo.Ver J. Díez Nicolás (1998), op. cit. [http:/www.oberaxemtas.upcomillas.es/Publicaciones/Libros/Actitudes/actitudes.htm] 


\section{Conclusiones}

En primer lugar, tenemos que destacar la necesidad metodológica de llevar a cabo estudios segmentados sobre los efectos de la inmigración como consecuencia de la desigual distribución geográfica de los inmigrantes, por lo que se instituye como imprescindible analizar la xenofobia a partir de la densidad de inmigración.

Aún existiendo limitaciones metodológicas para conciliar adecuadamente en este estudio la densidad de inmigrantes, los datos reflejan un apoyo a nuestra primera hipótesis, ya que su mayor número no parece ser determinante en el nivel de xenofobia existente, desde el momento que Cataluña, con más del doble de inmigrantes (13,48\%) que Andalucía (6,6\%), tiene un índice de xenofobia menor $(1,95)$ que Andalucía $(3,39)$, con una diferencia de 1,37 puntos en el año 2007.

En relación con la conclusión anterior, parece confirmarse nuestra segunda hipótesis, en el sentido que un mayor grado de modernización tiene efectos disolventes hacia la xenofobia. En efecto, la evolución de los índices de xenofobia desde 1991 hasta 2007 observan una tendencia de escaso nivel de xenofobia en ambas comunidades, aunque con un grado menor en Cataluña. No obstante, se observa un ligero aumento en los últimos años.

En segundo lugar, el perfil de los más y menos xenófobos en las dos comunidades tienen una alta coincidencia, a excepción de la identificación nacionalista y la posición social. Así, mientras en Cataluña los más xenófobos son los más nacionalistas, en Andalucía son los que se identifican por igual con su comunidad como con España, mientras que para los menos xenófobos la relación se invierte en ambas comunidades. Respecto a la posición social en Andalucía, cuanto más alta es, mayor es su grado de xenofobia, tendencia contraria de lo que ocurre en Cataluña, donde la menor posición social es la que genera más opiniones y conductas xenófobas.

En tercer lugar, se ha comprobado que los predictores de la xenofobia son muy parecidos en Andalucía y en Cataluña, de manera que los coincidentes son el nivel educativo, la ideología y la posición social, aunque para esta última variable, como hemos visto, tenga un comportamiento distinto en ambas comunidades.

Por último, los sentimientos hacia los diferentes grupos de inmigrantes parecen coincidir: son los árabes y musulmanes quienes concitan un mayor rechazo subjetivo. La mayor presencia de este colectivo, como su perceptiva complicación de integración, son las causas de su mayor rechazo. 


\section{Referencias bibliográficas}

BALIBAR, E. y Wallerstein, I. (1991). Raza, clase y nación. IEPALA.

BANTON, M. (1983). Racial and ethnic competition. Cambridge: Cambridge University Press.

BARKER, M. (1981). The new racism: Conservatives and the ideology of the tribe. Londres: Junction Book.

CARNERO RABAT, T. (1992). Modernización, desarrollo político y cambio social. Madrid: Alianza.

CASHDAN, E. (2001). «Ethocentrism and xenophobia a cross cultural study». Current Antropology, 42 (5), 760-765.

CEA D’AnCONA, M.A. (2004). La activación de la xenofobia en España. Madrid: CIS.

Colectivo IOÉ (2005). «Ciudadanos o intrusos: La opinión pública española ante los inmigrantes». Papeles de Economía Española, 104, 194-209.

El Mundo, 23 de mayo de 2008, 39.

DíEZ Nicolás, J. (1998). Actitudes hacia los inmigrantes. Madrid: Ministerio de Trabajo y Asuntos Sociales. Dirección General del Instituto de Migraciones y Servicios Sociales. [http:/www.oberaxemtas.upcomillas.es/Publicaciones/Libros/Actitudes/actitudes.htm]

- (2005). Las dos caras de la inmigración. Madrid: Ministerio de Trabajo y Asuntos Sociales.

EISENSTARDT, S.N. (1970). Ensayos sobre el cambio social y la modernización. Madrid: Tecnos.

GobERNADO ARribas, R. (1996). «Niveles educativos y estratificación social». En: Gobernado ARribas, R. (coord.). Análisis comparado de las estructuras sociales de Andalucía y Cataluña. Málaga: Universidad de Málaga y Universidad de Almería.

GONZÁlEZ ENRÍQUEZ, C. (2004). Opinión pública y politica de inmigración: Elementos de conflicto en la convivencia con los inmigrantes en España. Departamento de Ciencias Políticas y de la Administración, UNED, CPA Estudios. Working Papers.

GonZÁlez EnríQueZ, C. y Álvarez-MirandA, B. (2004). Inmigrantes en el barrio: Un estudio cualitativo de opinión pública. Madrid: Ministerio de Trabajo y Asuntos Sociales.

HECHTER, M. (1986). «Rational choice theory and the study of race and ethnic relations». En: Rex, J. y MASON, D. (eds.). Theory of race and ethnic relations. Cambridge: Cambridge University Press, 264-279.

HerranZ DE RAFAel, G. (2002). «La xenofobia en Andalucía y Cataluña: un análisis comparativo». En: La sociedad: Teoría e investigación. Madrid: CIS, 175-205.

- (2008). Xenofobia: un estudio comparativo en barrios y municipios almerienses. Madrid: CIS, 121, 107-132.

Hidalgo Tuñón, A. (1996). "Xenofobia». En: BlázQuez Ruiz, F.J. (dir.). 10 palabras clave sobre racismo y xenofobia. Navarra: Verbo Divino.

KaIN, J.F. (1965). "The effect of the ghetto on the distribution and level nonwhites employment in urban areas». En: Procedins. Social Statistic Section of the American Statical Association.

- (2004). «A pioneer's perspective on the Spatial Mismatch literature». Urban Studies, 41 (1), p. 7-32.

LUCAS, J. de (1992). Europa: ¿Convivir con la diferencia?: Racismo, nacionalismo y derechos de las minorías. Madrid: Tecnos. 
MaceDO, D. (2006). The globalization of racism. Boulder: Paradigm Publisher.

NuAMJOH, F. (2006). Insiders and outsiders: citizenship and xenophofia in contemporary southern Africa. Nueva York: Palgrave.

Pérez Yruela, M. y Desrues, T. (2005). Opinión de los españoles en materia de racismo y xenofobia. Madrid: Ministerio de Trabajo y Asuntos Sociales. Observatorio Español del Racismo y la Xenofobia.

Portes, A. y Rumbaut, R. (1996). Inmigrant in America. California: University of California Press.

Solé, C. (1991). Trabajadores extranjeros en Cataluña. ¿Integración o racismo? Madrid: CIS.

- (1976). Modernización: un análisis sociológico. Barcelona: Edicions 62.

- (1999). Modernidad y modernización. Barcelona: Anthropos.

TAguiefF, P.A. (1988). La force du préjugé: Essai sur le racisme et ses doubles. París: Le Découverte.

VAN DiJK, T.A. (2003). Ideología y discurso. Barcelona: Ariel.

Wieviorka, M. (1992). El espacio del racismo. Barcelona: Paidós.

WirTh, L. (1962). El urbanismo como forma de vida. Buenos Aires: Ediciones 3.

ZHOU, M. (1997). «Segmented assimilations: issues, controversias and recent research on the new second generation». International Migration Review, 31 (4), 975-1008. 